\title{
STRUCTURAL ANALYSIS BY DISTRIBUTION OF DEFORMATION*
}

\author{
BY \\ C. V. KLOUČEK \\ Prague
}

The external loading of any structure produces, on the one hand, deformation and on the other hand, stresses which spread from the source of deformation in all directions offered by the given structure; the bending moment may be regarded as a function of stress. The deformation in any arbitrary form, e.g. joint rotation, elongation, etc., represents in reality the single visible and measurable quantity. The expression of the external loading by means of fixed-end moments opened up the way for all the slope deflection solutions, as for instance, equations, balancing methods and the direct distribution of deformation. The balancing methods were based on the idea of alternately locking and unlocking the joints. The validity of both principles presented by Professors G. A. Maney and Hardy Cross follow from the fundamental law of super-position.

In the course of the original derivation of the D. of D.-system (see the author's earlier papers ${ }^{1}$ and books ${ }^{2}$ ), the direct moment distribution methods (Culman, Ritter, Suter etc.) were used as a pattern. Since these older computation systems are no longer currently known, the author here presents the derivation of the two basic D. of D.-relations using newer methods which are first of all briefly characterised.

Slope-deflection equations require first of all the establishment of all fixed-end moments $M^{\mathrm{fix}}$ on the assumption that all the joints of the given structure are fixed. After the simultaneous releasing of all joints, the sum of all the adjacent fixed-end moments acts as an impulse on the loaded joint. The deformation waves thus produced circulate through all joints until the complete settling-down of the loaded structure. The state of rest thus attained corresponds to the condition of equilibrium in every joint $\sum M=0$. If we substitute into this equation the basic relation between the bending moment $M_{s-k}$ and the joint rotations $\varphi$,

$$
M_{s-k}=\xi_{s-k}\left(2 \varphi_{s}+\varphi_{k}\right)+M_{s}^{\mathrm{fix}},
$$

then the slope deflection equation for the location-fixed joint $[s]$ and for $n$-adjacent joints $[k]$ can be written in the form

$$
\rho_{s} \varphi_{s}+\sum_{1}^{n}\left(\xi_{s-k} \varphi_{k}\right)=\sum M_{s}^{\mathrm{fix}} .
$$

The notation introduced here is the same as that used in the author's earlier publications:

$\xi_{s-k}=\xi_{k-s}$-denotes the stiffness factor of the member $[s]-[k]$ and has the value $I / L$.

*Received May 26, 1950

${ }^{1}$ Techn. Obzor, Prague, April 1938. Beton und Eisen, Berlin 1939, No. 24. Beton und Stahlbetonbau, Berlin 1943, Nos. 5, 6.

${ }^{2}$ Rozvod Deformace I, II, Stat. Naklad. Prague 1940, 1947; Das Prinzip der fortgeleiteten Verformung I, W. Ernst \& Sohn, Berlin, 1941; Distribution of deformation. A new method of structural analysis, C. V. Klouček, Prague, 1949, pp. 510, a limited English edition only for the information of foreign specialists. 
$\rho_{s}$-denotes the stiffness factor of the joint $[s]$ and equals twice the sum of all member factors, $\rho_{s}=2 \sum \xi_{s}$.

$\theta_{\mathrm{s}}$ - denotes the actual angular deformation of the joint [s].

$\varphi_{s}=2 E \theta_{s}$-denotes only a formal simplification for structures with constant modulus $E$.

The balancing methods of angle changes by Maney, Goldberg, Cotten, Grinter, Morris, Kammuller, etc. constitute in principle the deformation counterpart to the successive moment distribution method of Hardy Cross. The balancing system e.g. for a structure with three elastic joints may be carried out in various ways e.g. successively [1] - [2], [2] - [3] or throughout the whole structure [1] - [2] - [3], and the like. If the chosen successive system has to be exact, it must express the influence of every joint on every other joint. By means of the sum of the partial angular deformation in any one joint $[s]$, we obtain the resultant rotation $\varphi_{s}$,

$$
\varphi_{s}=\varphi_{s}^{x}+\varphi_{s}^{x x}+\varphi_{s}^{x x x}+\text { etc. }
$$

The magnitude of the individual increments $\varphi_{s}^{x}, \varphi_{s}^{x x}$, etc. is not a random quantity, but is governed by generally valid laws which can be expressed mathematically.

The direct D. of D.-Method was originally worked out as the deformation counterpart to the direct moment distribution method by E. Suter. ${ }^{3}$ The derivation could naturally

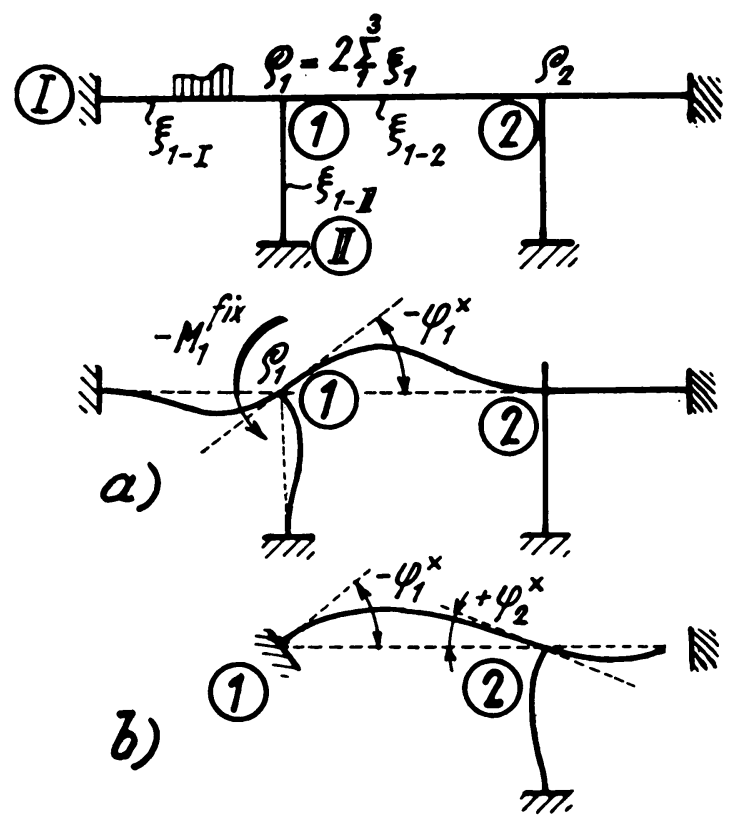

Fig. 1.

be worked out from any of the other exactly valid systems, e.g. according to Clapeyron. Here the two basic relations, for the resultant deformation of the loaded joint and for the direct deformation distribution to the adjacent joint, will be obtained by an analysis of 
the balancing principle. The individual deformation increments $\varphi_{s}^{x}, \varphi_{s}^{x x}$, etc., which were originally obtained by successive distribution there and back, will be expressed as resultant sums, thus avoiding the balancing system. The derived relations will be checked by means of methods working with resultant deformations $\varphi_{s}$ (e.g. by equations).

Solution for two elastic joints. For the case where a single span of the given structure is loaded (Fig. 1), the initial supposition that every $\varphi=0$ leads to the determination of the fixed-end moment $M_{1}^{\mathrm{fix}}$. The first stage of the balancing procedure, i.e. unlocking the loaded joint [1], is governed by the equation for one elastic joint $\rho_{1} \varphi^{x}=M_{1}^{\mathrm{fix}}$; the first partial deformation of the loaded joint [1] is therefore, according to Fig. 1a,

$$
\varphi_{1}^{x}=\frac{M_{1}^{\mathrm{fix}}}{\rho_{1}} .
$$

The second stage (Fig. 1b) assumes the locking of the joint [1] in the deformed position for $\varphi_{1}^{x}$ and the unlocking of joint [2]. The relation between the known, non-variable (fixed) value $\varphi_{1}^{x}$ and the unknown $\varphi_{2}^{x}$ is given by the single equation for the second joint $\rho_{2} \varphi_{2}^{x}+\xi_{1-2} \varphi_{1}^{x}=0$. The first partial deformation of the unloaded joint [2] is therefore

$$
\varphi_{2}^{x}=-\varphi_{1}^{x} \frac{\xi_{1-2}}{\rho_{2}} .
$$

After a further alternation of locking we obtain by backward distribution the second deformation increment in the first joint

$$
\varphi_{1}^{x x}=-\varphi_{2}^{x} \frac{\xi_{1-2}}{\rho_{1}} .
$$

Successive distribution (there and back) and addition of the corresponding partial values then gives the resultant deformations

$$
\begin{aligned}
& \varphi_{1}=\varphi_{1}^{x}+\varphi_{1}^{x x}+\varphi_{1}^{x x}+\text { etc. } \\
& \varphi_{2}=\varphi_{2}^{x}+\varphi_{2}^{x x}+\text { etc. }=-\varphi_{1} \frac{\xi_{1-2}}{\rho_{2}} .
\end{aligned}
$$

From these well-known balancing systems we can easily derive the direct D. of D.solution for the two elastic joints of Fig. 1. We express the second deformation increment $\varphi_{1}^{x x}$ of Eq. (6) in terms of the first deformation $\varphi_{1}^{x}$ of Eq. (5),

$$
\varphi_{1}^{x x}=\varphi_{1}^{x} \frac{\xi_{1-2}^{2}}{\rho_{1} \rho_{2}}=\alpha_{1-2} \varphi_{1}^{x},
$$

where the deformation constant $\alpha$ can arise only between two elastic joints, and its absolute value lies within the limits 0 and 0.25 . The next deformation increment $\varphi_{1}^{x x x}$ can be expressed by means of Eq. (9) as

$$
\varphi_{1}^{x x x}=\varphi_{1}^{x x} \frac{\xi_{1-2}^{2}}{\rho_{1} \rho_{2}}=\alpha_{1-2}^{2} \varphi_{1}^{x}
$$

and the resultant angle $\varphi_{1}$ at the loaded joint is obtained by substituting the partial deformations (9), (10), etc., into Eq. (7),

$$
\varphi_{1}=\varphi_{1}^{x}\left(1+\alpha_{1-2}+\alpha_{1-2}^{2}+a_{1-2}^{3}+\text { etc. }\right) .
$$


The deformation process between two elastic joints thus develops-in the same way as for moment distribution ${ }^{4}$-in the form of a simple geometrical series. On expressing the series (for $\alpha_{1-2}<1$ ) in the form

$$
\left(1+\sum_{i=1}^{i=\infty} \alpha_{1-2}^{i}\right)=\frac{1}{1-\alpha_{1-2}}
$$

and substituting for $\varphi_{1}^{x}$ from Eq. (4), we obtain the final expression for the loaded joint as in Fig. 1,

$$
\varphi_{1}=\frac{M_{1}^{\mathrm{fix}}}{\rho_{1}\left(1-\alpha_{1-2}\right)} .
$$

The direct distribution of this resultant deformation $\varphi_{1}$ to the unloaded joint [2] then has in this case the simplest form according to Eq. (8),

$$
\varphi_{2}=-\varphi_{1} \frac{\xi_{1-2}}{\rho_{2}} \text {. }
$$

Both the above expressions for $\varphi_{1}$ and $\varphi_{2}$ have been obtained by the addition of all the partial results from the completed balancing process [1] - [2]; the same expressions can be derived by elimination from the two slope-deflection equations. The sign of the individual angular deformations obtained by distribution, direct or successive, follows directly from the visual conception; joints which rotate in a clockwise direction are positive.

Solution for three elastic joints. The determination of the exact effect of a third joint opens up the way for the solution of every straight-beam structure and hence we devote more attention to this problem.

The above balancing system between two adjacent joints can be used successively also for three elastic joints as in Fig. 2. For the temporarily fixed joint [3], the balancing path [1]-[2] gives, according to Eqs. (12), (13) and (14),

$$
\begin{aligned}
& \varphi_{1}=\varphi_{1}^{x}\left(1+\sum_{i=1}^{i=\infty} \alpha_{1-2}^{i}\right)=\frac{M_{1}^{\mathrm{fix}}}{\rho_{1}\left(1-\alpha_{1-2}\right)}, \\
& \varphi_{2}^{x}=-\varphi_{1} \frac{\xi_{1-2}}{\rho_{2}} .
\end{aligned}
$$

For the fixed joint [1], the successive distribution [2] - [3] of the partial deformation $\varphi_{x}^{2}$ gives the resultant $\varphi_{2}=\varphi_{2}^{x}+\varphi_{2}^{x x}$,

$$
\varphi_{2}=\varphi_{2}^{x}\left(1+\sum_{i=1}^{i-\infty} \alpha_{2-3}^{i}\right),
$$

which after simplification and introduction of $\varphi_{1}$ from Eq. (16) gives

$$
\varphi_{2}=-\varphi_{1} \frac{\xi_{1-2}}{\rho_{2}\left(1-\alpha_{2-3}\right)} .
$$

The resultant deformation of the third joint is obtained from the known relation

$$
\varphi_{3}=-\varphi_{2} \frac{\xi_{2-3}}{\rho_{3}} .
$$

${ }^{4}$ See e.g. Professor H. Cross, Trans. ASCE, 96, pp. 147, 150, 1932. 
When using only the two successive distributions [1] - [2] and [2] - [3], the whole balancing process is not yet at an end; from Fig. 2 it is clear that for instance, the effect

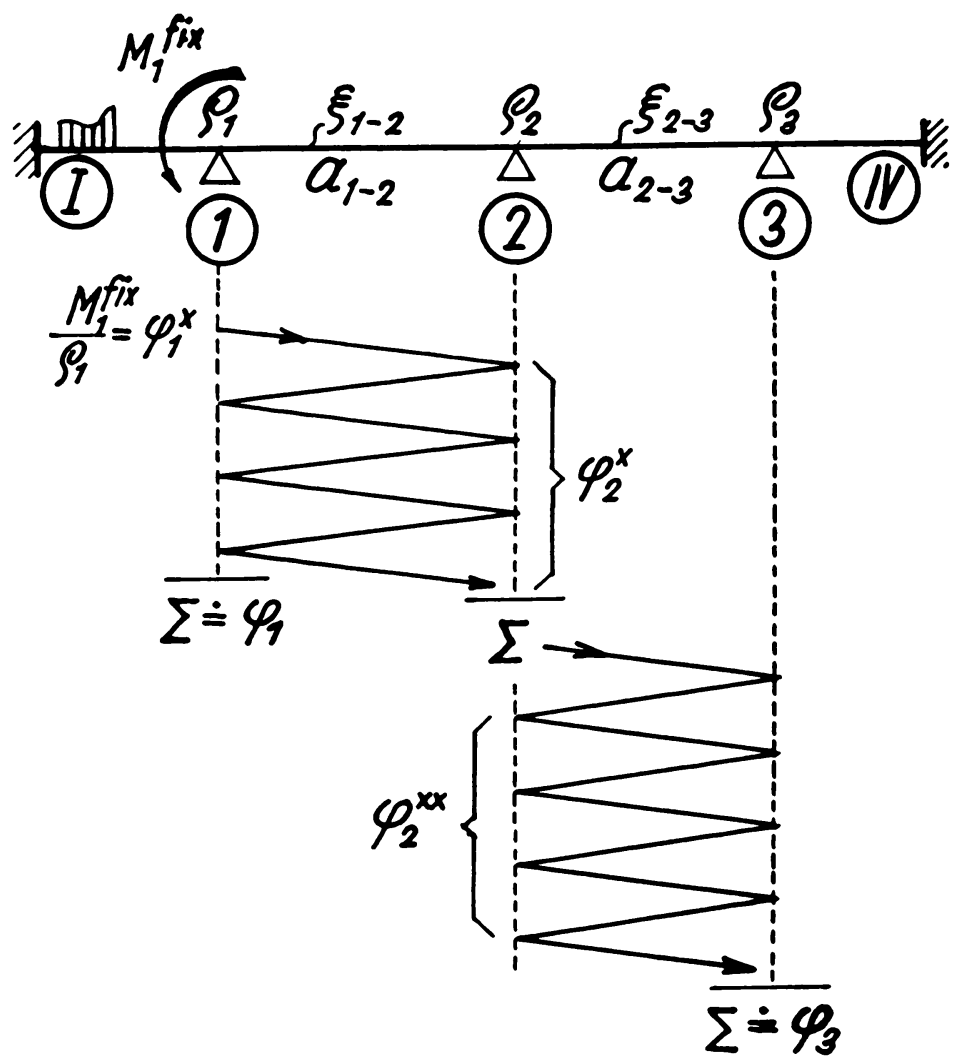

FIG. 2.

of unlocking joint [3] on joint [1] has not been expressed, i.e. that all possible deformation paths according to Fig. 4 have not yet been pursued. Both described successive and direct

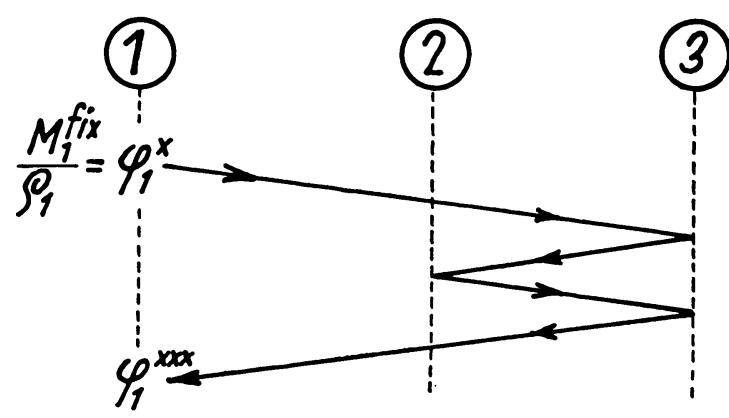

Fig. 3.

solutions cannot exceed a maximum error of $2 \%$ for three elastic joints and are therefore well suited for the solution of practical problems; the incomplete balancing process naturally cannot be used for the determination of the exact effect of the third joint. 
The creative idea of locking and unlocking the joints can be used in any arbitrary sequence, where the simplest form of the carry-over factor $\xi / \rho$ is successively introduced for the corresponding span. The successive distribution, e.g. in the row $1-2-3-2-3-$ $2-1$ as in Fig. 3 can be expressed in terms of the constants $\alpha$,

$$
\varphi_{1}^{x x}=\varphi_{1}^{x} \frac{\xi_{1-2} \xi_{2-3} \xi_{3-2} \xi_{2-3} \xi_{3-2} \xi_{2-1}}{\rho_{2} \rho_{3} \rho_{2} \rho_{3} \rho_{2} \rho_{1}}=\varphi_{1}^{x} \alpha_{1-2} \alpha_{2-3}^{2} .
$$

In Fig. 4 there are indicated all the distribution paths for three elastic joints which give for example in the loaded joint $\varphi_{1}=\varphi_{1}^{x}+\varphi_{1}^{x x}+$ etc. The evaluation of the first

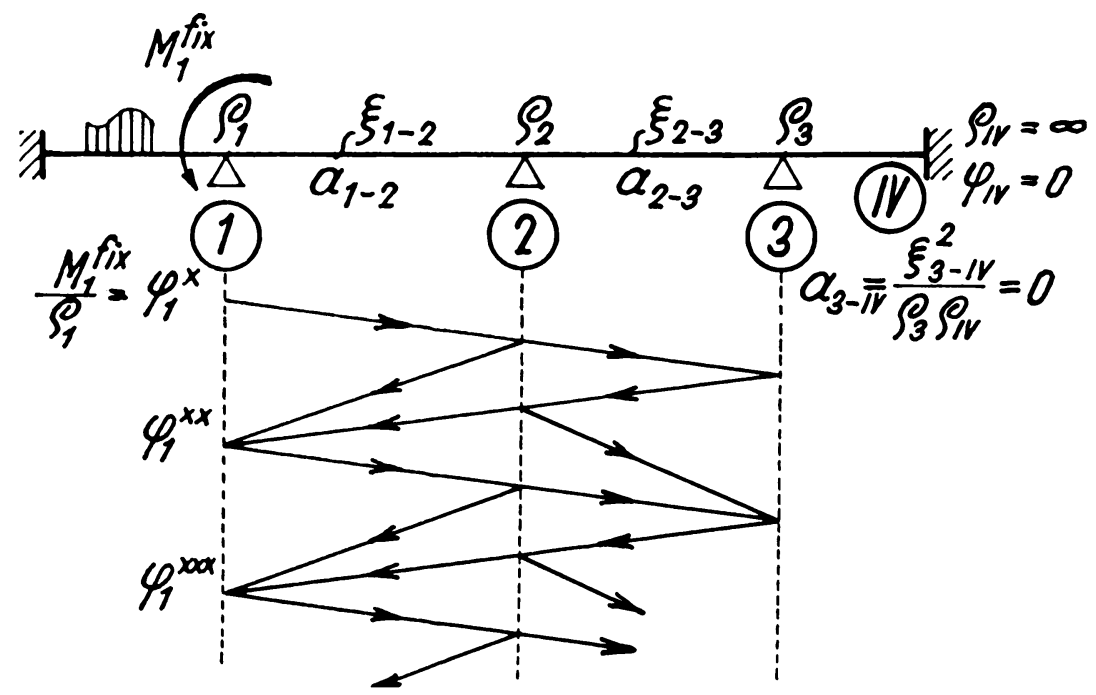

FIg. 4.

deformation increment $\varphi_{1}^{x x}$ is possible by only two paths $1-2-1$ and $1-2-3-2-1$, which, expressed in the sense of Eq. (20), gives

$$
\varphi_{1}^{x x}=\varphi_{1}^{x}\left(\alpha_{1-2}+\alpha_{1-2} \alpha_{2-3}\right) .
$$

Then the first rough approximation for $\varphi_{1} \doteq \varphi_{1}^{x}+\varphi_{1}^{x x}$ can be written in the form

$$
\varphi_{1} \doteq \frac{M_{1}^{\mathrm{fix}}}{\rho_{1}}\left\{1+\alpha_{1-2}\left(1+\alpha_{2-3}\right)\right\}
$$

which is in agreement with the exact expression (27) given below on substituting for both indices $i=1$. In a similar way we could obtain further partial increments in the joint [1] and could derive the final expression for $\varphi_{1}$. This procedure would be exact but considerably time-consuming; the same may be said of the balancing system $1-2-3-2-1-2-$ etc. according to Table 1.

All the balancing systems for three elastic joints indicated here are only of schematic nature, since they take no account of the various intervals of time required by individual deformation waves. On the assumption that the balancing process is continued until the complete settling-down of the given structure $(i=\infty)$, it is naturally immaterial in 
what order we add together the individual deformation increments $\varphi=\varphi^{x}+\varphi^{x x}+$ etc. While maintaining the basic condition that the effect of every joint on every other joint should be expressed, we can choose the balancing system shown in Fig. 5 .

\begin{tabular}{|c|c|c|c|c|c|}
\hline يٌ & $-\varphi_{1}$ & $\begin{array}{l}\text { corry- } \\
\text { over } \\
\text { factor }\end{array}$ & $+\varphi_{2}$ & $\begin{array}{l}\text { carry- } \\
\text { over } \\
\text { factor }\end{array}$ & $-\varphi_{3}$ \\
\hline 1. & $\begin{array}{l}\varphi_{1}^{x} \\
\varphi_{1}^{x x}\end{array}$ & $\begin{array}{l}\times \frac{\xi_{1-2}}{\rho_{2}} \\
-\frac{\xi_{2-1}}{\rho_{1}} \times\end{array}$ & $\begin{aligned}- & \varphi_{2}^{x} \\
\varphi_{2}^{x x} & \\
& \left(\varphi_{2}^{x}+\varphi_{2}^{x x}\right)\end{aligned}$ & $\frac{\xi_{2-3}}{\rho_{3}} \frac{\xi_{3-2}}{\rho_{2}} \times$ & $\begin{array}{l}-\varphi_{3}^{x} \\
\left(\varphi_{3}^{x}\right)\end{array}$ \\
\hline 2. & $\left(\varphi_{1}^{x x}\right)$ & $\begin{array}{l}\times \frac{\xi_{1-2}}{\rho_{2}} \\
-\frac{\xi_{2-1}}{\rho_{1}} \times\end{array}$ & $\begin{array}{c}-\varphi_{2}^{x x x} \\
\left(\varphi_{2}^{x+x}+\varphi_{2}^{x x x}\right) \\
\varphi_{2}^{x \times x x}= \\
\left(\varphi_{2}^{n x+x}+\varphi_{2}^{x \times x x}\right)\end{array}$ & $\begin{array}{l}\frac{\xi_{2-3}}{\rho_{3}} \\
\frac{\xi_{3-2}}{\rho_{2}} \times\end{array}$ & $\begin{array}{c}-\varphi_{3}^{\mathrm{xx}} \\
\left(\varphi_{3}^{\mathrm{xx}}\right)\end{array}$ \\
\hline 3. & $\left(\varphi_{1}^{x+x}\right)$ & $\times \frac{\xi_{1-2}}{\rho_{2}}$ & etc. & & \\
\hline
\end{tabular}

Making use of Maney's principle (every $\varphi=0$ ) we obtain the fixed-end moment $M_{1}^{\mathrm{fix}}$ and the first value $\varphi_{1}^{x}$. On using the simple carry-over factor $\xi / \rho$, the unlocking of joint [2] gives

$$
\varphi_{2}^{x}=-\varphi_{1}^{x} \frac{\xi_{1-2}}{\rho_{2}} .
$$

Completing the balancing of the value $\varphi_{2}^{x}$ between joints [2] - [3] gives according to Eq. (17) the result $\varphi_{2}^{x}\left(1+\sum \alpha_{2-3}^{i}\right)$. After substituting $\varphi_{1}^{x}$ for $\varphi_{2}^{x}$ from the above equation (23), the fixing of joint [2] and unfixing of joint [1] gives the first increment in the loaded joint

$$
\begin{aligned}
\varphi_{1}^{x x} & =\varphi_{1}^{x} \frac{\xi_{1-2}}{\rho_{2}}\left(1+\sum \alpha_{2-3}^{i}\right) \frac{\xi_{2-1}}{\rho_{1}} \\
& =\varphi_{1}^{x} \alpha_{1-2}\left(1+\sum \alpha_{2-3}^{i}\right) .
\end{aligned}
$$

In the same way the second and third cycles give

$$
\begin{aligned}
\varphi_{1}^{x x x} & =\varphi_{1}^{x x} \alpha_{1-2}\left(1+\sum \alpha_{2-3}^{i}\right) \\
& =\varphi_{1}^{x}\left[\alpha_{1-2}\left(1+\sum \alpha_{2-3}^{i}\right)\right]^{2} \\
\varphi_{1}^{x x x x} & =\varphi_{1}^{x}\left[\alpha_{1-2}\left(1+\sum \alpha_{2-3}^{i}\right)\right]^{3} .
\end{aligned}
$$


The exact value of the resultant deformation follows from the sum $\varphi_{1}=\varphi_{1}^{x}+\varphi_{1}^{x x}+$ etc. according to Fig. 5,

$$
\varphi_{1}=\frac{M_{1}^{\mathrm{fix}}}{\rho_{1}}\left\{1+\sum_{i=1}^{i=\infty}\left[\alpha_{1-2}\left(1+\sum_{i=1}^{i=\infty} \alpha_{2-3}^{i}\right)\right]^{i}\right\} .
$$

The balancing process for the case of three elastic joints thus obeys the law of a geometri-

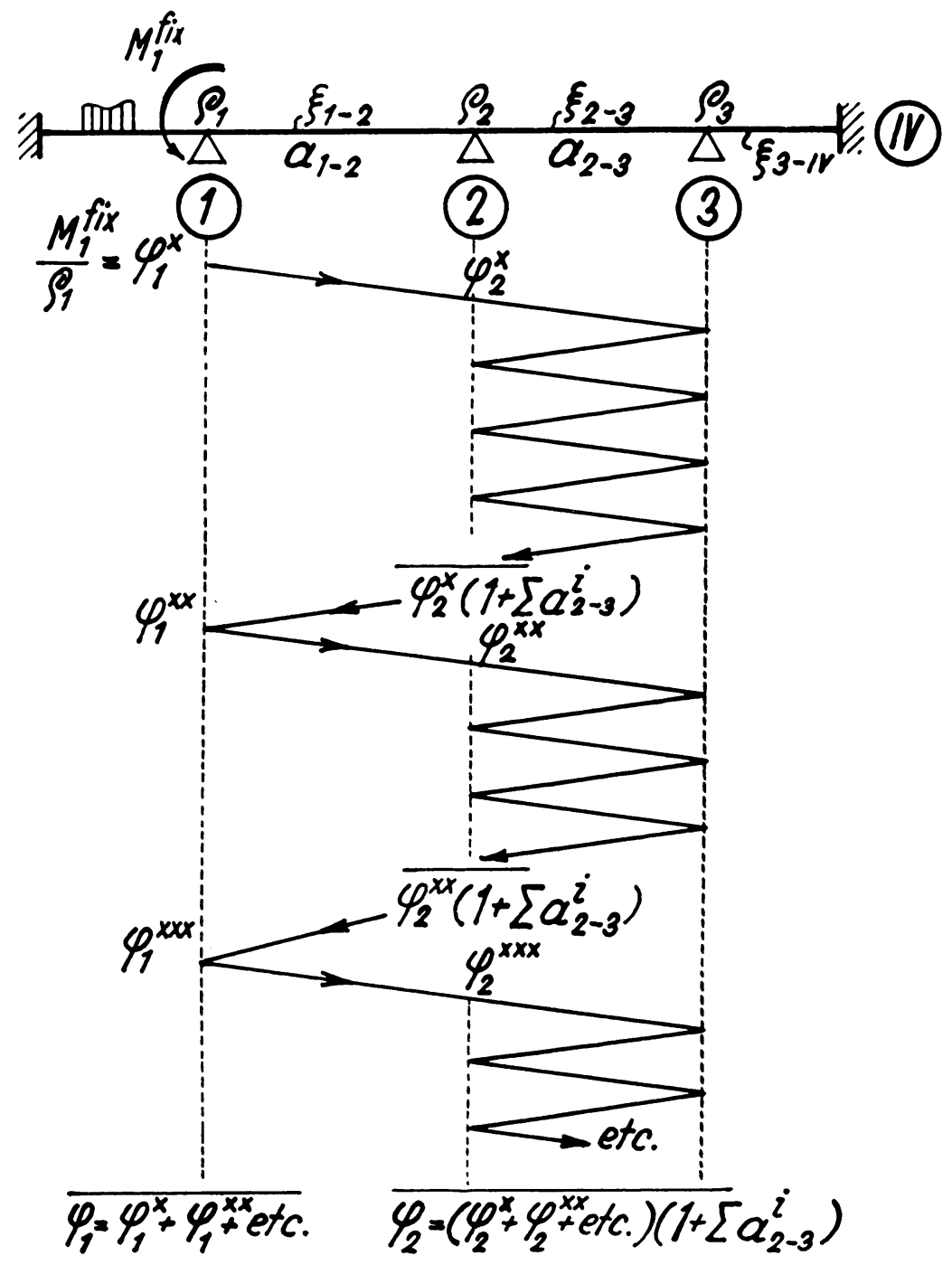

Fig. 5.

cal series of higher order i.e. a series of a series. After expressing the inner series in finite form, the outer series can be expressed in the form

$$
1+\sum_{i=1}^{i=\infty}\left[\frac{\alpha_{1-2}}{1-\alpha_{2-3}}\right]^{i}=\frac{1}{1-\alpha_{1-2} /\left(1-\alpha_{2-3}\right)} .
$$


The exact value for the loaded joint is thus given by the simple expression

$$
\varphi_{1}=\frac{M_{1}^{\mathrm{fix}}}{\rho_{1}\left[1-\alpha_{1-2} /\left(1-\alpha_{2-3}\right)\right]} .
$$

By addition of the partial values according to Fig. 5, we obtain the exact result for the distributed deformations

$$
\begin{aligned}
& \hat{c}_{1}=-\varphi_{1} \frac{\xi_{1-2}}{\rho_{2}\left(1-\alpha_{2-3}\right)}, \\
& \varphi_{3}=-\varphi_{2} \frac{\xi_{2-3}}{\rho_{3}},
\end{aligned}
$$

which differ from the previous expressions (18) and (19) only by the exact value $\varphi_{1}$.

The results (29) to (31) can easily be checked by any other method derived from the final stage of the balancing process, i.e. from the condition of equilibrium $\sum M=0$. Using the elimination method ${ }^{5}$ of Professor J. B. Wilbur, we have for $M_{3-\mathrm{IV}}=-M_{3-2}$, according to Fig. 5,

$$
\begin{aligned}
& \xi_{3-\mathrm{IV}}\left(2 \varphi_{3}\right)+\xi_{2-3}\left(2 \varphi_{3}+\varphi_{2}\right)=0 \\
& \varphi_{3}=-\varphi_{2} \frac{\xi_{2-3}}{2\left(\xi_{2-3}+\xi_{3-\mathrm{IV}}\right)}=-\varphi_{2} \frac{\xi_{2-3}}{\rho_{3}} .
\end{aligned}
$$

The condition of equilibrium $\sum M_{2}=0$ for the joint [2], expressed in terms of deformations

$$
\xi_{1-2}\left(2 \varphi_{2}+\varphi_{1}\right)+\xi_{2-3}\left(2 \varphi_{2}-\varphi_{2} \frac{\xi_{2-3}}{\rho_{3}}\right)=0
$$

gives, after rearrangement and for $\xi_{2-3}^{2} / \rho_{2} \rho_{3}=\alpha_{2-3}$,

$$
\varphi_{2}=-\varphi_{1} \frac{\xi_{1-2}}{\rho_{2}\left(1-\alpha_{2-3}\right)} .
$$

The condition of equilibrium $M_{1-\mathrm{I}}+M_{1-2}=M^{\mathrm{fix}}$ expressed in terms of deformations

$$
\xi_{\mathrm{I}-1}\left(2 \varphi_{1}\right)+\xi_{1-2}\left[2 \varphi_{1}-\varphi_{1} \frac{\xi_{1-2}}{\rho_{2}\left(1-\alpha_{2-3}\right)}\right]=M_{1}^{\mathrm{fix}}
$$

gives, after introducing the constant $\alpha_{1-2}$, the known expression (29),

$$
\varphi_{1}=\frac{M_{1}^{\mathrm{fix}}}{\rho_{1}\left[1-\alpha_{1-2} /\left(1-\alpha_{2-3}\right)\right]} .
$$

If for the structure of Fig. 5, we introduce an elastic support at the fourth point (denoted [4] instead of [IV]), the deformation of the loaded joint [1] proceeds according to a geometrical series of the third order. Successive introduction of the finite limits for the individual series results in a continued (chain) fraction, which we denote by the chain value $\alpha^{\prime}$,

$$
\varphi_{1}=\frac{M_{1}^{\mathrm{fix}}}{\rho_{1}\left(1-\alpha_{1-2}^{\prime}\right)}=\frac{M_{1}^{\mathrm{fix}}}{\rho_{1}\left\{1-\alpha_{1-2} /\left[1-\alpha_{2-3} /\left(1-\alpha_{3-4}\right)\right]\right\}} .
$$

${ }^{5}$ Transactions A.S.C.E., 102, p. 346 (1937). 
In the same way the first deformation distribution to the joint [2] will be

$$
\varphi_{2}=-\varphi_{1} \frac{\xi_{1-2}}{\rho_{2}\left(1-\alpha_{2-3}^{\prime}\right)}=-\varphi_{1} \frac{\xi_{1-2}}{\rho_{2}\left[1-\alpha_{2-3} /\left(1-\alpha_{3-4}\right)\right]} .
$$

For the case where the central joint [2] is loaded, we can evaluate the exact value $\varphi_{2}$ by the sum of two balancing systems A and B as shown in Fig. 6. The simple distribution

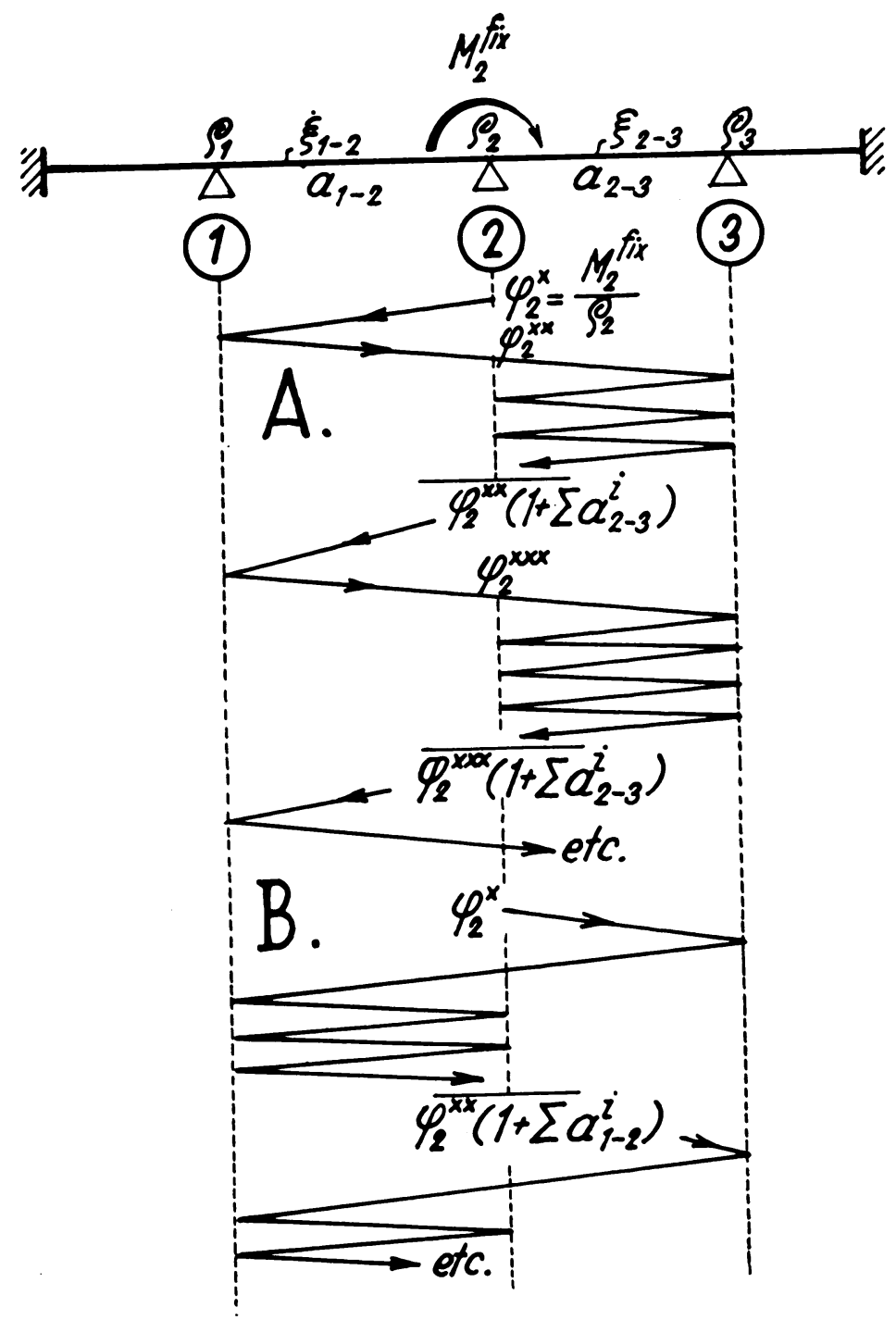

Fig. 6.

[2] - [1] - [2] of the value $\varphi_{2}^{x}$ gives $\varphi_{2}^{x x}=\varphi_{2}^{x} \alpha_{1-2}$ and after completing the balancing [2] - [3] of the value $\varphi_{2}^{x x}$, we have the first increase

$$
\varphi_{2}^{x x}\left(1+\sum \alpha_{2-3}^{i}\right)=\varphi_{2}^{x} \alpha_{1-2}\left(1+\sum \alpha_{2-8}^{i}\right),
$$


and similarly

$$
\varphi_{2}^{x x x}\left(1+\sum \alpha_{2-3}^{i}\right)=\varphi_{2}^{x}\left[\alpha_{1-2}\left(1+\sum \alpha_{2-3}^{i}\right)\right]^{2} .
$$

On interchanging the subscripts, we obtain an analogical expression for the balancing system B. The resultant deformation $\varphi_{2}$ is obtained by the summation of two geometrical series of second order,

$$
\varphi_{2}=\varphi_{2}^{x}\left\{1+\sum_{i=1}^{i=\infty}\left[\alpha_{1-2}\left(1+\sum_{i=1}^{i-\infty} \alpha_{2-3}^{i}\right)\right]^{i}+\sum_{i=1}^{i-\infty}\left[\alpha_{2-3}\left(1+\sum_{i=1}^{i-\infty} \alpha_{1-2}^{i}\right)\right]^{i}\right\} .
$$

When we write all the series in finite form and rearrange the terms, ${ }^{6}$ we obtain as the deformation of the central joint [2] the simple expression

$$
\varphi_{2}=\frac{M_{2}^{\mathrm{fix}}}{\rho_{2}\left(1-\alpha_{1-2}-\alpha_{2-3}\right)} .
$$

This relation can be easily checked by means of slope-deflection equations. If we substitute for the two unknowns from the first and third equations

$$
\begin{aligned}
& \varphi_{1}=-\varphi_{2} \frac{\xi_{1-2}}{\rho_{1}} \\
& \varphi_{3}=-\varphi_{2} \frac{\xi_{2-3}}{\rho_{3}}
\end{aligned}
$$

into the second equation $\rho_{2} \varphi_{2}+\xi_{1-2} \varphi_{1}+\xi_{2-3} \varphi_{3}=M_{2}^{\mathrm{fix}}$, the latter may be written in the form

$$
\varphi_{2}=\frac{M_{2}^{\mathrm{fix}}}{\rho_{2}\left(1-\xi_{1-2}^{2} / \rho_{1} \rho_{2}-\xi_{2-3}^{2} / \rho_{2} \rho_{3}\right)} .
$$

For more complex open structures such as that shown in Fig. 7, we obtain by elimina-

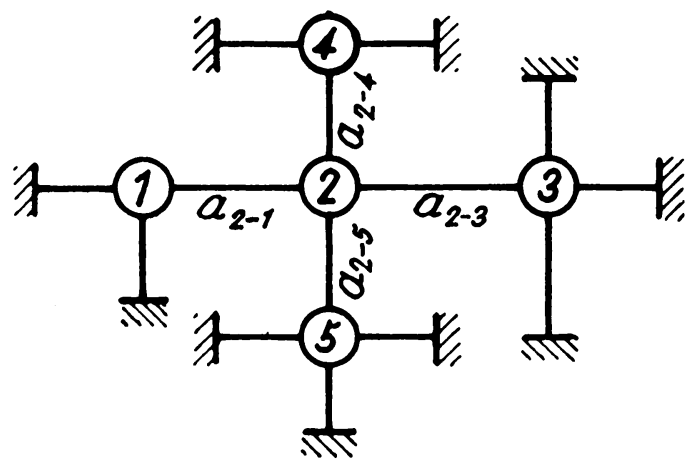

FIG. 7.

tion, in the manner of Eqs. (39) and (40), the expression for the loaded central joint [2],

$$
\varphi_{2}=\frac{M_{2}^{\mathrm{fix}}}{\rho_{2}\left(1-\sum_{1}^{4} \alpha_{2}\right)},
$$

${ }^{6}$ See Distribution of Deformation, pp. 26, 31, 32. 
which is identical with the approximate formula derived by Mr. C. A. Willson, M.ASCE for a frame network. ${ }^{7}$

For the loading of the outer joint [3] of Fig. 7, we have

$$
\varphi_{3}=\frac{M_{3}^{\mathrm{fix}}}{\rho_{3}\left(1-\alpha_{3-2}^{\prime}\right)} \quad \text { where } \quad \alpha_{3-2}^{\prime}=\frac{\alpha_{3-2}}{1-\alpha_{2-1}-\alpha_{2-4}-\alpha_{2-5}},
$$

and for the fixed joint [3], i.e. for $\alpha_{2-3}=0$, the distribution of the known (non-variable) deformation $\varphi_{3}$ to the joint [2] gives

$$
\varphi_{2}=-\varphi_{3} \frac{\xi_{3-2}}{\rho_{2}\left(1-\alpha_{2-1}-\alpha_{2-4}-\alpha_{2-5}\right)} .
$$

If we summarise now the results of the foregoing analysis, we see that every straightbeam structure can be solved by means of two generally valid relations for the loaded joint $[s]$,

$$
\varphi_{s}=\frac{\sum M_{s}^{\mathrm{fix}}}{\rho_{s}\left(1-\sum_{1}^{n} \alpha_{s}^{\prime}\right)}
$$

and for the direct (resultant) deformation distribution to the adjacent joint [ $k]$.

$$
\varphi_{k}=-\varphi_{s} \frac{\xi_{s-k}}{\rho_{k}\left(1-\sum_{1}^{n-1} \alpha_{k}^{\prime}\right)},
$$

where $n$ denotes the number of elastic members meeting in the joint under consideration.

In concluding this paper, the author would like to add the following few remarks:

This article has been devoted only to the theoretical analysis of the basic relations. The solutions for simultaneous loading, closed structures, joint displacement, etc. are given in earlier publications. ${ }^{2}$

The author's attention has been drawn several times to the fact that the solution of linear slope-deflection equations by means of continued fractions is of certain interest from a purely mathematical point of view; the author has not gone into this problem in detail but doubts if the mathematical applicability of the $\mathrm{D}$ of D.-system will be so general as, for instance, the Relaxation Method by Professor R. Southwell.

The limited English edition of the work "Distribution of Deformation" has been printed only for specialists abroad; several copies of the book are in various American libraries (e.g. Brown University). The author intends to use the valuable information obtained through contact with specialists in various countries either for the working out of a comparative study or as an addition for the standard edition of the D. of D.-book where also the appropriate literature will be listed.

${ }^{7}$ Transactions A.S.C.E., 102, pp. 352-354 (1937). 